\title{
Multimedia Standards. History. State of Art ${ }^{\star}$
}

\author{
Peter L. Stanchev ${ }^{1,2}$ \\ 1 Institute of Mathematics and Informatics \\ Bulgarian Academy of Sciences, Sofia, Bulgaria \\ 2 Kettering University, Flint, USA \\ pstanche@kettering.edu
}

\begin{abstract}
The aim of this presentation is to review some of the standards, connected with multimedia and their metadata. We start with MPEG family and continue with Open Standards for Interactive TV. Efficient video-streaming is presented. Some standards for audiovisual metadata are outline. We finish with W3C standards.
\end{abstract}

Keywords: Multimedia Standards, MPEG, Interactive TV, VideoStream Filtering, Audiovisual Media, W3C.

\section{MPEG Standards}

The Moving Picture Experts Group (MPEG) [6] is a working group of ISO/IEC in charge of the development of standards for coded representation of digital audio and video and related data. Since 1988 when it has been established, the group has produced standards that help the industry offer end users an ever more enjoyable digital media experience. The number of independent standards is more than 125. Follow is a list of standard families.

- MPEG-1, the standard for such products as Video CD and MP3 are based;

- MPEG-2, the standard for such products as Digital Television set top boxes and DVD are based;

- MPEG-4, the standard for multimedia for the fixed and mobile web;

- MPEG-7, the standard for description and search of audio and visual content;

- MPEG-21, the Multimedia Framework;

- MPEG-A, the standard for application-specific formats;

- MPEG-B, a collection of Systems specific standards;

- MPEG-C, a collection of Video specific standards;

- MPEG-D, a collection of Audio specific standards;

- MPEG-DASH, the standard for video streaming over the internet;

- MPEG-E, a standard (M3W) providing support to download and execution of multimedia applications;

* This work was supported in part by Open Access Infrastructure for Research in Europe (OpenAIRE) EU project, and the Bulgarian National Science Fund under the Project D002 308 "Automated Metadata Generating for e-Documents Specifications and Standards".

T.-h. Kim et al. (Eds.): FGIT 2011, LNCS 7105, pp. 39-42 2011.

(C) Springer-Verlag Berlin Heidelberg 2011 
- MPEG-H, a standard (HEVC) that will provide significantly increased video compression performance compared to AVC;

- MPEG-M, a standard (MXM) for packaging and reusability of MPEG technologies;

- MPEG-U, a standard for rich-media user interface;

- MPEG-V, a standard for interchange with virtual worlds.

In its 23 years of activity MPEG has developed an impressive portfolio of technologies that have created an industry worth several hundred billion USD. In the moment in the full development phase are the following standards:

- 3D Video Coding, the standard for coding 3D Visual information;

- High Efficiency Video Coding, the standard for a new frontier in video coding;

- MPEG-M 2nd edition, the standard for digital media ecosystems.

An example of MPEG-4 system is the ANIMATION system - a system for animation scene and contents creation, retrieval and display [9]. Semantic Video and Image Retrieval is analyzed in [10].

The upcoming Reconfigurable Video Coding (RVC) standard currently developed at MPEG supports the construction of video standards as libraries of coding tools. These libraries can be incrementally updated and extended, and the tools in them can be aggregated to form complete codecs using a streaming programming model, which preserves the inherent parallelism of the coding algorithm [4].

Depending on the specific characteristics of an image data set, some features can be more effective than others when performing similarity search. Starting from this observation, a technique that predicts the effectiveness of MPEG 7 image features based on a statistical analysis of the specific data sets in the Multimedia Content Management System was developed [8].

MPEG-V (ISO/IEC 23005) provide an architecture and associated information representations to enable the interoperability between virtual worlds, e.g., digital content provider of a virtual world, gaming, simulation, DVD, and with the real world, e.g., sensors, actuators, vision and rendering, robotics, support for independent living, social and welfare systems, banking, insurance, travel, real estate, rights management and many others [2].

\section{Open Standards for Interactive TV}

Levels of interactivity TV [7] can be described as: Level 1 - Basic TV, Level 2 Call-In-TV, Level 3 - Parallel TV, Level 4 - Additive TV, Level 5 - Service on Demand, Communicative TV, and Level 7 - Fully Interactive TV. The Multimedia and Hypermedia Information Coding Expert Group (MHEG) [5], a subgroup of the International Organization for Standardization (ISO), published the MHEG standards. A digital video broadcaster filtering algorithm is presented in [1]. 


\section{Standards in the Audiovisual Media}

The main issues in the Audiovisual Media standards for audio visual media include [3]:

- EBU P Meta. The European Broadcasting Union (EBU) has defined P Meta as a metadata vocabulary for program exchange in the professional broadcast industry;

- Material Exchange Format. The Material Exchange Format (MXF) is a standard issued by Society of Motion Picture and Television Engineers (SMPTE), defining the specification of a file format for the wrapping and transport of essence and metadata in a single container.

- SMPTE Descriptive Metadata Scheme 1 (DMS-1), formerly known as Geneva Scheme uses metadata sets defined in the SMPTE Metadata Dictionary;

- SMPTE Metadata Dictionary (is a large thematically structured list of narrowly defined metadata elements, defined by a key, the size of the value and its semantics;

- Standard Media Exchange Framework (SMEF) the data model defined by the $\mathrm{BBC}$ to describe the metadata related to media items (media objects) and programs and parts thereof (editorial objects), down to the shot level;

- Controlled Vocabulary and Ontologies. Audiovisual content descriptions often contain references to semantic entities such as objects, events, states, places, and times.

\section{W3C Standards}

The World Wide Web Consortium (W3C) [11] develops technical specifications and guidelines through a process designed to maximize consensus about the content of a technical report, to ensure high technical and editorial quality, and to earn endorsement by W3C and the broader community. They include:

- Web Design and Applications involve the standards for building and rendering Web pages, including HTML5, CSS, SVG, Ajax, and other technologies for Web Applications;

- Web Architecture focuses on the foundation technologies and principles which sustain the Web, including URIs and HTTP;

- Semantic Web technologies enable people to create data stores on the Web, build vocabularies, and write rules for handling data. Linked data are empowered by technologies such as RDF, SPARQL, OWL, and SKOS;

- XML Technologies including XML, XQuery, XML Schema, XSLT, XSL-FO, Efficient XML Interchange (EXI), and other related standards;

- Web of Services refers to message-based design frequently found on the Web and in enterprise software. The Web of Services is based on technologies such as HTTP, XML, SOAP, WSDL, SPARQL, and others; 
- Web of devices. This includes Web access from mobile phones and other mobile devices as well as use of Web technology in consumer electronics, printers, interactive television, and even automobiles;

- Browsing and authoring tools. Web agents are intended to serve users.

\section{Conclusion}

The aim of the multimedia standards are to enable transparent and augmented use of multimedia resources across a wide range of networks, devices, user preferences, and communities, notably for trading of content.

\section{References}

1. Falchi, F., Gennaro, C., Savino, P., Stanchev, P.: Efficient Video Stream Filtering. IEEE Multimedia, 52-61 (January-March 2008)

2. Gelissen, J.: Introduction to MPEG-V. Journal of Virtual Worlds Research, Technology, Economy, and Standards 2(3), 4-7 (2009)

3. ISO/IEC 14496-11, Information technology, Coding of audio-visual objects, Part 11: Scene description and application engine, http://www.iso.org/iso/iso_catalogue/catalogue_tc/ catalogue_detail.htm?csnumber $=54839$

4. Janneck, J., Mattavelli, M., Raulet, M., Wipliez, M.: Reconfigurable Video Coding - a Stream Programming Approach to the Specification of New Video Coding Standards. In: MMSys 2010, February 22-23, pp. 223-234 (2010)

5. MHEG organization, http://www.mheg.org/users/mheg/index.php

6. MPEG organization, http://www.mpeg.org/

7. Ruhrmann, G., Nieland, J.: Interaktives Fernsehen. Westdeutscher Verlag GmbH, Wiesbaden (1997)

8. Stanchev, P., Amato, G., Falchi, F., Gennaro, C., Rabitti, F., Savino, P.: Selection of MPEG-7 Image Features for Improving Image Similarity Search on Specific Data Sets. In: 7th IASTED International Conference on Computer Graphics and Imaging, CGIM 2004, Kauai, Hawaii, pp. 395-400 (2004)

9. Stanchev, P.: ANIMATION, System for Animation Scene and Content Creation, Retrieving and Viewing. In: SPIE, vol. 4672, pp. 86-94 (2002)

10. Stanchev, P.: Semantic Video and Image Retrieval - State of the Art and Challenges. In: ACMSE 2007, The 45th ACM Southeast Conference, Winston-Salem, North Carolina, USA, March 24 (2007)

11. W3C organization, http://www.w3.org/standards/ 